\title{
Laser thermal ablation of multidrug-resistant bacteria using functionalized gold nanoparticles
}

\author{
This article was published in the following Dove Press journal: \\ International Journal of Nanomedicine \\ 23 March 2017 \\ Number of times this article has been viewed
}

\section{Lucian Mocan ${ }^{1,2}$ \\ Flaviu A Tabaran ${ }^{3}$ \\ Teodora Mocan ${ }^{2,4}$ \\ Teodora Pop 5 \\ Ofelia Mosteanu ${ }^{5}$ \\ Lucia Agoston-Coldea ${ }^{6}$ \\ Cristian T Matea $^{2}$ \\ Diana Gonciar ${ }^{2}$ \\ Claudiu Zdrehus ${ }^{1,2}$ \\ Cornel lancu'}

'3rd Department of General Surgery, "Iuliu Hatieganu" University of

Medicine and Pharmacy, ${ }^{2}$ Department of Nanomedicine, "Octavian

Fodor" Gastroenterology Institute, ${ }^{3}$ Department of Pathology, University of Agricultural Sciences and Veterinary Medicine, Faculty of Veterinary

Medicine, ${ }^{4}$ Department of Physiology,

${ }^{5} 3 r d$ Gastroenterology Department, ${ }^{6}$ Department of Internal Medicine,

"Iuliu Hatieganu" University of

Medicine and Pharmacy, Cluj-Napoca, Romania
Correspondence: Lucian Mocan;

Cornel lancu

3rd Surgery Clinic, "Iuliu Hatieganu"

University of Medicine and Pharmacy,

19-21 Croitorilor Street, Cluj-Napoca

400238, Romania

Tel/fax +40 264439696

Email mocanlucian@yahoo.com;

cornel.iancu@umfcluj.ro

\begin{abstract}
The issue of multidrug resistance (MDR) has become an increasing threat to public health. One alternative strategy against MDR bacteria would be to construct therapeutic vectors capable of physically damaging these microorganisms. Gold nanoparticles hold great promise for the development of such therapeutic agents, since the nanoparticles exhibit impressive properties, of which the most important is the ability to convert light into heat. This property has scientific significance since is exploited to develop nano-photothermal vectors to destroy bacteria at a molecular level. The present paper summarizes the latest advancements in the field of nanotargeted laser hyperthermia of MDR bacteria mediated by gold nanoparticles.
\end{abstract}

Keywords: bacteria, photo-thermal ablation, gold nanoparticles, antibiotic resistance

\section{Introduction}

The problem of multidrug resistance (MDR) has become a growing threat to public health. A high number of infections and many deaths that occur each year in the USA are caused by bacteria that resist multiple antibiotics. ${ }^{1-7}$ Another issue is the rejection of certain drugs, a recent trend that has intensified the urgency of developing new therapeutic agents and approaches. Current studies are investigating the possible benefits of natural products, the changes that might be brought to certain classes of antibiotics in use today, and the production of host defense peptides. ${ }^{1,8-11}$ Due to their physical properties and potencies, nanoparticles have proven to be highly versatile and can be widely used in numerous applications. ${ }^{6,12-14}$ Their size and shape properties are compatible with various biomedical applications that require interaction with smallmolecule antibiotics. The size of these nanomaterials provides a large surface-areato-volume ratio, which enables the binding of a large number of high affinity ligands, allowing for nanoparticle multivalency in fighting bacteria. Based on their features, nanoparticles have been functionalized with antibiotics and used as antibacterial agents. Loading can be achieved by both covalent and noncovalent interactions. The resulting complexes have shown greater antibacterial effect and lower minimum inhibitory concentration than the antibiotics alone. This efficiency is the result of the polyvalent impact caused by the concentration of antibiotics on the surface of the nanoparticles, which is associated with a significant improvement in the amount of internalization of antibiotics by nanoparticles. The conjugation of functional ligands onto the surface of gold nanoparticles (GNPs) allows for direct multivalent interactions, which demonstrates the application of these nanomaterials as self-therapeutics. Such an approach could negate the drawbacks of the encapsulation of current antimicrobials. As they exhibit low toxicity and chemical inertia, GNPs have proven ideal for the assembly of self-therapeutics. GNPs have gained significant interest as nanovectors for therapy, 
representing a novel and competent substitute for effective transportation and translocation of therapeutic molecules. ${ }^{15-22}$ Various types of GNP complexes (protein-coated GNPs, peptide-coated GNPs, GNPs coated with nucleic acids, drugs) are available for delivery to cells and organs.

One of the most remarkable properties of GNPs is the ability to transform light into heat under laser irradiation. ${ }^{2,6,18,23-26}$ This property is significant because it can be exploited to develop nano-photothermal vectors in order to destroy bacteria at a molecular level. The present paper summarizes the latest advancements in the field of nanotargeted laser hyperthermia of MDR bacteria mediated by GNPs (Table 1).

\section{Nano-photothermal therapy mediated by GNPs in MDR Staphylococcus aureus Gold nanoparticles}

Light-absorbing GNPs have been functionalized with specific antibodies to achieve targeted and selective laser bacterial killing. ${ }^{27}$ This therapeutic intervention was assessed in real time using photothermal microscopy. The antibacterial effect was caused by the combination of strong overheating in the laser impact zone and the generation of bubbles around gold clusters. These results were confirmed by photothermal imaging and time-resolved imaging. The technology was further employed to selectively kill $S$. aureus by means of targeted delivery of 10, 20, and $40 \mathrm{~nm}$ GNPs functionalized with anti-protein A antibodies. Following exposure to laser pulses (420-570 nm, $12 \mathrm{~ns}, 0.1-5 \mathrm{~J} / \mathrm{cm}^{2}, 100$ pulses), several

Table I Antibacterial activity against multidrug-resistant bacteria correlated with the type of gold nanoparticles used

\begin{tabular}{lll}
\hline $\begin{array}{l}\text { Antibacterial activity } \\
\text { against }\end{array}$ & $\begin{array}{l}\text { Type of gold } \\
\text { nanoparticles }\end{array}$ & $\begin{array}{l}\text { Reference } \\
\text { no }\end{array}$ \\
\hline Staphylococcus aureus & Gold nanospheres & 27,28 \\
& Gold nanocages & 29 \\
& Gold nanorods & $30-32$ \\
& Gold-silver core-shell & 33 \\
& nanoparticles & \\
Pseudomonas aeruginosa & Gold nanostars & 34,35 \\
& Gold nanocrosses & 36 \\
Salmonella & Gold nanorods & 37 \\
Enterococcus faecalis & Popcorn-shaped gold & 38,39 \\
Escherichia coli & nanoparticles & \\
& Gold nanoshell & 40 \\
& Gold nanorod/hollow SiO & 42 \\
& Gold nanorods & 43 \\
\hline
\end{tabular}

techniques such as electron microscopy, optical transmission, and viability testing assessed laser killing of bacteria for different laser fluences and nanoparticle size ranges. ${ }^{27}$

In a study testing the antibacterial effect resulting from the association between antibody-targeted GNPs and pulsed laser irradiation, ${ }^{28}$ GNPs were functionalized with $S$. aureus-specific antibodies, followed by methicillin-resistant $S$. aureus (MRSA) and methicillinsensitive $S$. aureus incubation. Suspensions were further irradiated with a pulsed laser $(8 \mathrm{~ns}, 532 \mathrm{~nm}$, fluence range $\left.1-5 \mathrm{~J} / \mathrm{cm}^{2}\right)$. The change in effect caused by this type of exposure was assessed at fluences ranging from 0 to $5 \mathrm{~J} / \mathrm{cm}^{2}$. The results obtained in the group exposed to fluences of $0 \mathrm{~J} / \mathrm{cm}^{2}$ showed a survival rate of $86 \% \pm 20 \%$ (mean $\pm \mathrm{SD}$, $\mathrm{n}=6$ ) compared to controls, with a decrease in survival from $75 \% \pm 11 \%$ to $31 \% \pm 8 \%$ in subjects exposed to fluences of $5 \mathrm{~J} / \mathrm{cm}^{2}$. After exposure to 2,3 , and $5 \mathrm{~J} / \mathrm{cm}^{2}$, there was a significant decrease in survival when compared to the first group, while differences were not as considerable for fluences of $1 \mathrm{~J} / \mathrm{cm}^{2}$. Estimations made using regression analysis indicated evidence of a linear relationship between the destruction of drug-resistant bacteria by photothermal therapy and laser beam fluence $\left(r^{2}=0.97\right)$. The use of ligandfree nanoparticles or the absence of laser irradiation led to a considerably lower antibacterial effect (Figure 1).

\section{Gold nanocages}

$S$. aureus was used as a proof-of-principle ESKAPE (Enterococcus faecium, S. aureus, Klebsiella pneumoniae, Acinetobacter baumannii, Pseudomonas aeruginosa, and Enterobacter species) pathogen in a recent study ${ }^{29}$ to show that the loading of the correct antibiotic, such as daptomycin, into the polydopamine coating based on gold nanocages (AuNC@PDA) - where the strategy adopted for their synthesis was galvanic replacement - resulted in an outer edge length of $55 \pm 5 \mathrm{~nm}$ and an inner edge length of $38 \pm 5 \mathrm{~nm}$. Another finding was that the conjugation of these complexes to antibodies targeting $S$. aureus surface protein A can selectively and directly deliver the nanoscale constructs onto the bacterial cell surfaces, as seen in Figure 1. The fact that there was no binding in mammalian cells helped confirm target specificity. The effect of $808 \mathrm{~nm}$ diode laser irradiation on AuNC@PDA (200 $\mu \mathrm{L}, 0.04-0.4$ nM) was represented by colony forming units (CFU) reduction below detection limits (20 CFU/well) at 0 hour. However, 24 hours after treatment, the cells were able to rebound above baseline. On the other hand, there was a decrease in bacterial cell viability below detection limits at 0 and 24 hours after laser irradiation of 
A

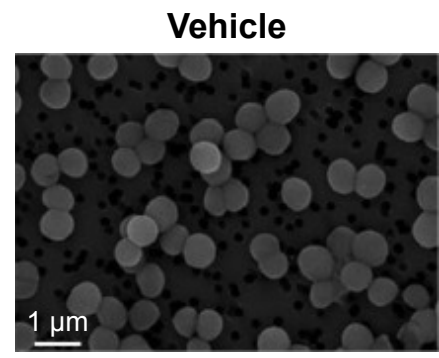

GNPs

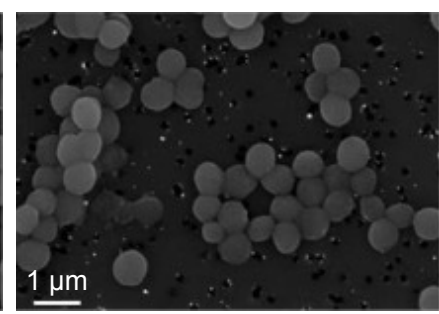

fGNPs

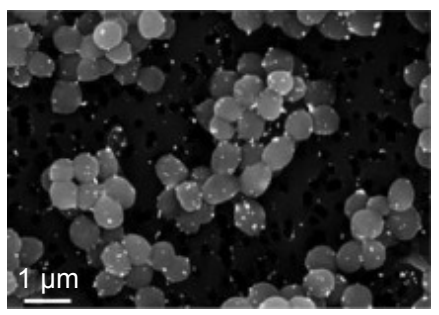

B
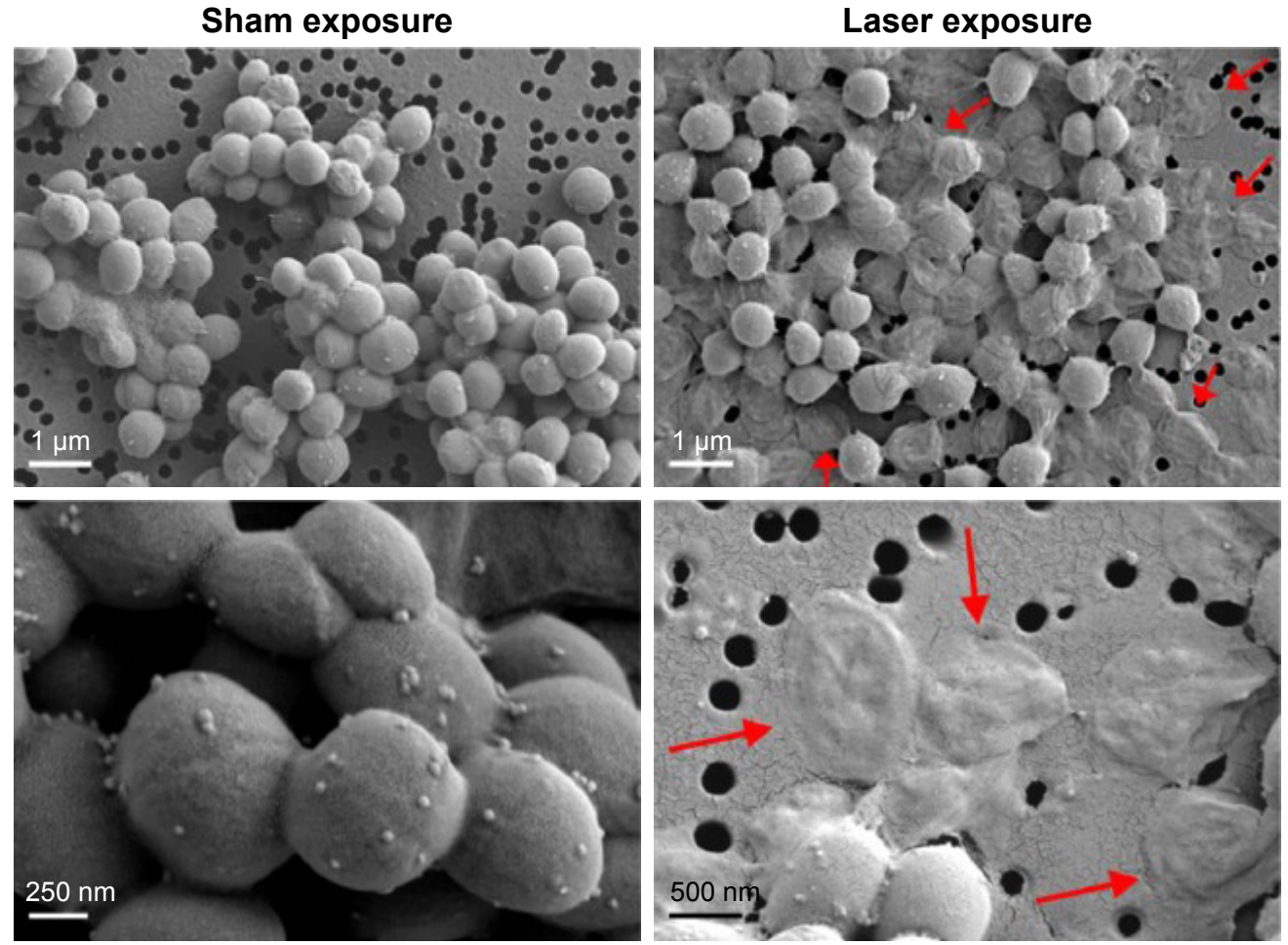

Figure I Scanning electron microscope images of MSSA.

Notes: (A) Backscattered electron images of MSSA treated with PBG vehicle, nonfunctionalized GNPs, or fGNPs. (B) Secondary electron images showing MSSA treated with fGNPs and then sham exposed or exposed to pulsed laser irradiation at $532 \mathrm{~nm}$. Red arrows indicate damaged bacterial cells. Copyright (C) 2015 . Dove Medical Press. Reproduced from Millenbaugh NJ, Baskin JB, DeSilva MN, Elliot WR, Glickman RD. Photothermal killing of Staphylococcus aureus using antibody-targeted gold nanoparticles. Int J Nanomedicine. 2015;10:1953-1960.28

Abbreviations: fGNPs, functionalized GNPs; GNPs, gold nanoparticles; MSSA, methicillin-sensitive Staphylococcus aureus; PBG, phosphate buffered saline containing I\% bovine serum albumin and $10 \%$ glycerol.

$4 \mu \mathrm{g} / \mathrm{mL}$ AuNC@DapLo/PDA (polydopamine-coated gold nanocages) ${ }^{29}$ There was also evidence of a decrease in the antibacterial effects of antibiotics in the presence of unconjugated aSpa depending on the concentration of AuNC@Dap/ PDA-aSpa nanoscale constructs. Therefore, the authors were able to confirm target specificity and greater synergistic antibacterial efficiency by means of bacterial cell surface localization of AuNC@Dap/PDA-aSpa instead of the more abstract effects of microtiter plate-based antibacterial assays.

\section{Gold nanorods}

An agent that can detect MRSA and selectively destroy it when used in combination with photothermal therapy was developed in another study ${ }^{30}$ using gold nanorods (GNRs) and an anti-protein A antibody. The authors synthesized GNRs functionalized with polystyrene sulfonate, binding antiprotein A antibody to their surface. The preparation of the final complex was confirmed by Fourier transform infrared spectroscopy and ultraviolet-visible spectra. After the colonies were counted, the results indicated greater cell death $(82 \%)$ in groups undergoing treatment with the experimental nanoconjugate in association with photothermal therapy than in others $(P<0.05)$. After treatment with free GNRs combined with near-infrared (NIR) laser therapy, bacterial cell death (16\%) was not significantly greater than in controls $(P<0.05)$. Moreover, no cell death occurred when using NIR laser therapy alone. The extent to which the GNRs conjugated to anti-protein A antibody were absorbed in the vital organs 
of infected BALB/c mice at time intervals of $1,4,8$, and 24 hours following intravenous inoculation was expressed as the percentage of injected dose per gram of organ $(\% \mathrm{ID} / \mathrm{g})$. Outcomes revealed that the nanocomplex accumulated at the site of infection to a greater extent than in the respective healthy tissues and organs at all time intervals and especially, at 8 hours after inoculation $(P<0.05)$. A considerable decrease in bacterial viability $(73 \%)$ was observed in the in vivo murine models following photothermal therapy. ${ }^{30}$

GNRs conjugated to toluidine blue photosensitizer (Au-anionic poly(acrylic acid)-toluidine blue) showed its duality in a study conducted by Kuo et al on MRSA, determining both antimicrobial photodynamic inactivation and hyperthermia. ${ }^{31}$ Following treatment with the abovementioned conjugate and sequential exposure to heliumneon laser (633 nm, 1 minute) and continuous wave laser (808 nm, 25 minutes), there was a significant reduction in MRSA viability (to about 5\%) and a 10-fold increase in antimicrobial ability, compared to photodynamic antimicrobial chemotherapy or hyperthermia alone. There was no bacterial damage in the absence of nanorods after both types of laser therapies, but there was a decrease in viability to $59 \%$ and $53 \%$ following treatment with nanorods. ${ }^{31}$

Effective biofilm disinfection was obtained in another study ${ }^{32}$ using a GNR cluster that is easily excited by NIR laser irradiation, which generates heat rapidly through photothermal conversion. Wet chemistry was used for the synthesis of GNRs, and photolithography was used in the microfabrication of one GNR cluster. After being directly applied to the biofilm, the influence of the photothermal effect of the GNR cluster on the biofilm was assessed both prior to laser irradiation and after treatment, which showed a significant decrease in cell viability and biofilm thickness. As indicated by analysis of bacterial mortality rates and biofilm inactivation, greater efficacy was observed for longer exposures to laser irradiation. Clear morphologic damage was indicated by scanning electron microscopy images of bacteria following irradiation, including bacterial cell membrane rupture.

\section{Core-shell gold-silver nanoparticles}

Aspartame-stabilized gold-silver nanostructures (AuNP@) $\mathrm{Ag} @$ Asm) with core-shell architecture were developed in an attempt to associate the antimicrobial activity of silver with the thermosensitive properties of gold (Figure 2). ${ }^{33}$ Broth microdilution antibacterial assay was used to determine minimum inhibitory concentrations. The experiment was performed on strains of $S$. aureus subsp. aureus Rosenbach



Figure 2 Gold-silver core-shell nanoparticles stabilized with aspartame proposed by Fasciani et al. ${ }^{33}$

Notes: Reprinted with permission from Fasciani C, Silvero MJ, Anghel MA, Argüello GA, Becerra MC, Scaiano JC. Aspartame-stabilized gold-silver bimetallic biocompatible nanostructures with plasmonic photothermal properties, antibacterial activity, and long-term stability. J Am Chem Soc. 20I4;136(50):17394-17397. Copyright (C) 2014 American Chemical Society. ${ }^{33}$

Abbreviation: AuNP@Ag@Aspartame, aspartame-stabilized gold-silver nanostructures.

(ATCC 25923), Staphylococcus epidermidis SE19, and Escherichia coli CF073. Experiments on 120 identical plates kept in the dark or exposed to LED irradiation (530 $\mathrm{nm}$ ) and stored at a temperature of $37^{\circ} \mathrm{C}$ for 18 hours were carried out in parallel to determine the optical density of the bacterial cultures. Experiments were performed with and without irradiation therapy to assess the antibacterial effect of AuNP@Ag@Asm and the photothermal effect of gold. Results showed that bacterial killing was not only incomplete in the dark (even for below-detection values) but was also followed by regrowth. The opposite result was obtained following irradiation. Biocompatibility testing was performed using the MTS cell proliferation colorimetric assay, in which any significant effect on the proliferation of primary dermal fibroblasts following treatment with AuNP@Ag@Asm at different concentrations was not observed. ${ }^{33}$

\section{Gold nanostars}

In a recent study, ${ }^{34}$ monolayers of gold nanostars were synthesized by lauryl sulfobetaine-driven seeded growth method. The anti-biofilm activity was evaluated by preparing 32 round glass slides $(1.0 \mathrm{~cm}$ in diameter $)$ using localized surface plasmon resonance spectroscopy (802 nm, s=12) and setting the surface concentration of the adsorbate on the gold surface to $3.0 \mathrm{mg} / \mathrm{cm}^{2}$ ( $\left.\mathrm{s}=0.4\right)$. Experiments were carried out using a strong biofilm producer, an MRSA LP strain. Following laser irradiation, the conjugate exhibited an excellent 


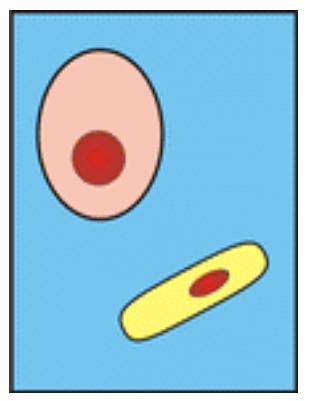

Bacterial cell or other pathogen

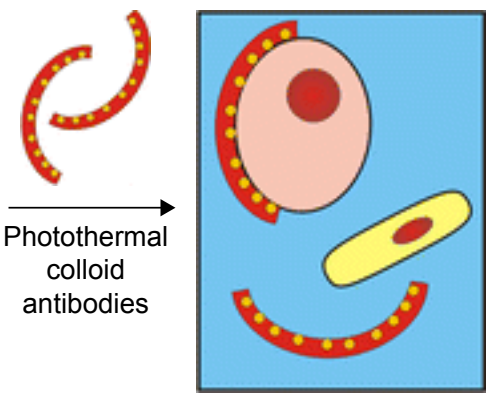

Cell shape recognition

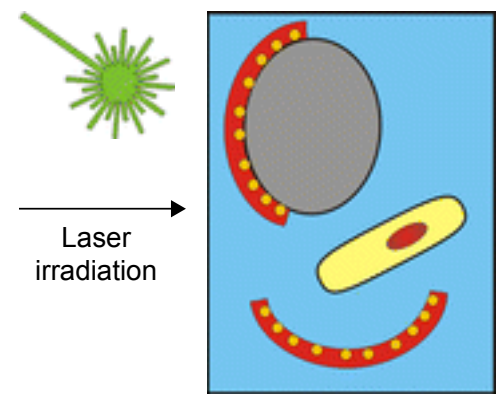

Shape-selective killing of bacteria

Figure 3 Proposed mechanism for shape-selective recognition and killing of microorganisms by Borovička et al. ${ }^{35}$

Notes: Reprinted with permission from Borovička J, Metheringham WJ, Madden LA, Walton CD, Stoyanov SD, Paunov VN. Photothermal colloid antibodies for shapeselective recognition and killing of microorganisms. J Am Chem Soc. 20I3; I35(I4):5282-5285. Copyright (C) 2013 American Chemical Society. ${ }^{35}$

photothermal conversion property in the NIR region. After exposure to $808 \mathrm{~nm}$ laser, the monolayers of gold nanostars generated a rise in tumor temperature (hyperthermia), which resulted in the efficient killing of the $S$. aureus biofilm. ${ }^{34}$

A class of shape- and size-selective antimicrobial agents was developed in a study conducted by Borovička et al..$^{35}$ The experiment consisted of the fabrication of imprinted colloid particles using cell templates by the fragmentation of silica shells. The increased contact area between the target cell and the colloidal imprint led to the mechanism of recognition, which can help develop "colloid antibodies" for efficient shape-selective binding (Figure 3). GNPs were integrated into the silica shell of the colloid imprint to bind to matching yeast cells, which was followed by direct delivery onto the target cell surface. Laser irradiation of the cell dispersion prompted selective killing of the target cells. The impact of the photothermal colloid antibody on shape-selective recognition and killing of bacteria was also confirmed by a mixture of two differently shaped and differently sized types of bacterial cells. ${ }^{35}$

\section{Nano-photothermal therapy mediated by GNPs in MDR $P$. aeruginosa \\ Gold nanorods}

Covalent binding of primary antibodies to GNRs was used for the selective destruction of $P$. aeruginosa (Figure 4). The synthesis of nanorods for bacterial cell wall-targeted delivery was achieved by electrostatic or covalent binding of anti-PA3 primary antibodies to their surfaces. Bacteria were incubated with relevant antibody-nanorod complexes. Evidence of electron-dense GNR-covered bacteria was offered by transmission electron microscopy. Live and dead cell counts using the Live/Dead kit (Invitrogen, Carlsbad, CA, USA) revealed up to $80 \%$ cell viability in the case of PA3 cells without the attachment of nanorods or NIR irradiation, NIR-irradiated cells without the attachment of nanorods, and bacteria with attached nanorods and without NIR irradiation. A $75 \%$ decrease in cell viability was observed following NIR irradiation of nanorod-coated PA3 cells, along with a considerable increase in the number of dead or compromised cells $(P<0.05)$. According to these findings, the functionalization of GNRs with antibodies and their exposure to NIR light determined the selective targeting of PA3 cells. Therefore, these complexes are able to reduce cell viability to a great extent. ${ }^{36}$

\section{Gold nanocrosses}

The characterization of high NIR absorbing multibranched gold nanocrosses $(\sim 35 \mathrm{~nm})$ was presented by other authors. ${ }^{37}$ Antibody-functionalized gold nanocrosses were used for targeting PcrV, a protein involved in the translocation of type III secreted toxins of $P$. aeruginosa. The bacterial suspension of $P$. aeruginosa PAO1 was tested in the presence of the conjugates using confocal fluorescence microscopy to assess the effect produced by the photoexcitation of gold nanocrosses with strong NIR absorption. The experiment consisted of the following steps: the preparation of $500 \mu \mathrm{L}$ PAO1 bacterial suspension $\left(1 \times 10^{8}\right.$ cells $/$ $\mathrm{mL}$ ), followed by incubation with antibody-functionalized gold nanocrosses for 1 hour (final concentration $0.1 \mathrm{mg}$ / $\mathrm{mL}$ ). Following the washing step, the effect produced by photoexcitation at $800 \mathrm{~nm}$ (power density of $\approx 3.0 \mathrm{~W} / \mathrm{cm}$ ) for 5 minutes was assessed using confocal microscopy. Total destruction of $P$. aeruginosa was accomplished by the nanocomplex following NIR irradiation therapy, with 

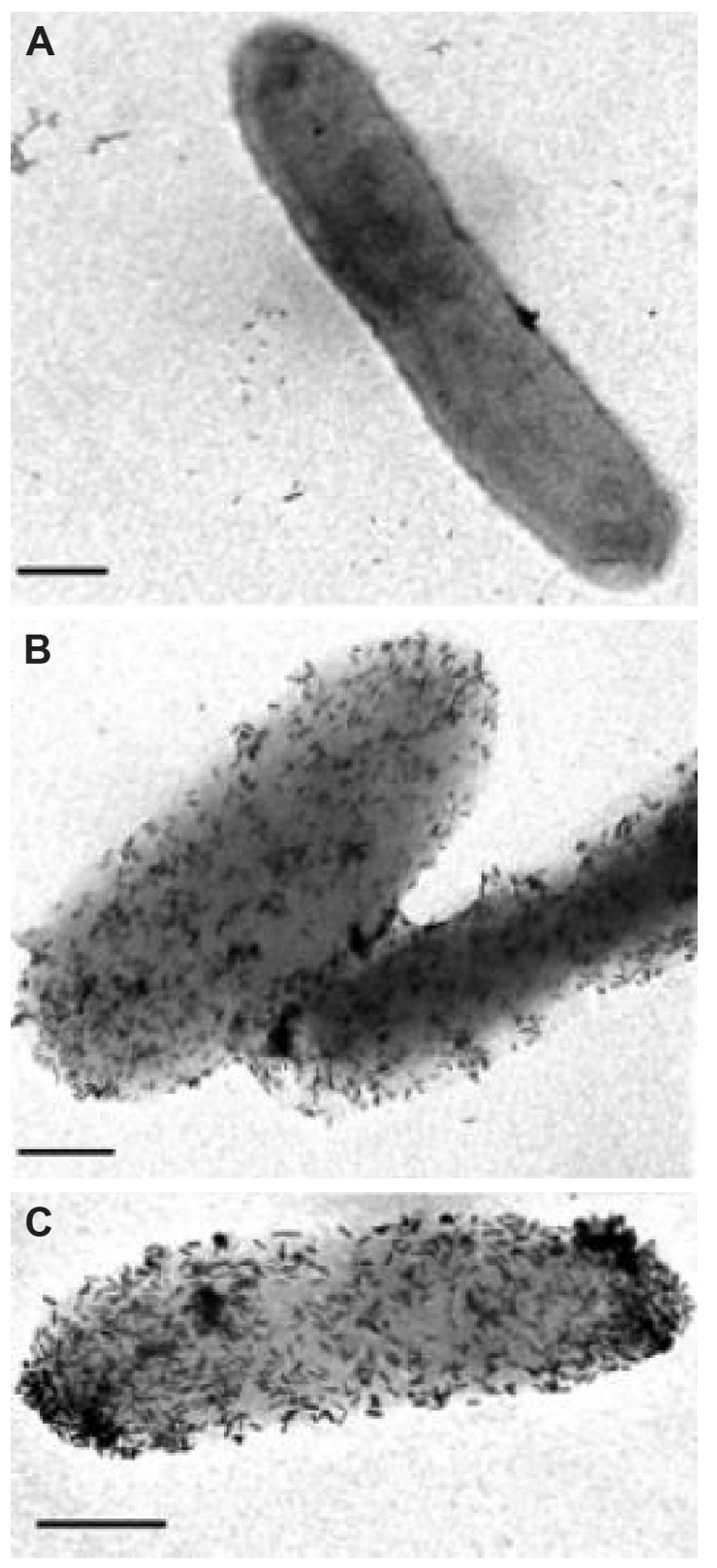

Figure 4 Transmission electron microscopy images (magnification: 30,000x) representing PA3 - antibody-nanorod conjugates interactions.

Notes: (A) PA3 - ligand-free nanorods; (B) PA3-coated antibody-nanorod conjugates (electrostatic attraction); and (C) PA3-coated antibody-nanorod conjugates (covalent coupling using I-ethyl-3-(3-dimethyl aminopropyl)-carbodiimide chemistry). Scale bar $=500 \mathrm{~nm}$. Reprinted with permission from Norman RS, Stone JW, Gole A, Murphy CJ, Sabo-Attwood TL. Targeted photothermal lysis of the pathogenic bacteria, pseudomonas aeruginosa, with gold nanorods. Nano lett. 2008;8(I):302-306. Copyright @ 2008, American Chemical Society. ${ }^{36}$

the absence of bacterial activity at 48 hours after treatment. This confirms the effective photothermal killing of bacteria and the prevention of bacterial regrowth. These gold nanocross conjugates proved to be successful for effectively targeted and localized photothermal ablation of MDR bacteria, reducing the risk or severity of damage to normal tissue. ${ }^{37}$

\section{Nano-photothermal therapy mediated by GNPs in MDR Salmonella bacteria}

In their study, Lin and Hamme developed an assay for the quick and accurate detection of bacteria by measuring the affinity binding of antibodies, the two-step, seed-mediated synthesis of popcorn-shaped GNPs (GNPop), the use of surface-enhanced Raman scattering, and the introduction of the inductively coupled plasma mass spectrometry analytical technique. ${ }^{38} \mathrm{~A}$ qualitative assessment indicated the presence of Salmonella in 10 minutes using Raman spectroscopy, while 100 isolates of Salmonella typhimurium definitive type $10^{4}$ were detected in 40 minutes in $1 \mathrm{~mL}(100 \mathrm{CFU} / \mathrm{mL})$ during a quantitative assessment. Concentration-dependent $(\mathrm{CFU} / \mathrm{mL})$ laser therapy $\left(1 \mathrm{~W} / \mathrm{cm}^{2}, 670 \mathrm{~nm}\right)$ indicated the strong relationship between this type of treatment and the killing of Salmonella. Photothermal damage achieved by the conjugation of monoclonal M3038 antibody to GNPop resulted in the killing of nearly $100 \%$ of the bacteria (Salmonella, $10^{5} \mathrm{CFU} / \mathrm{mL}$ ) in 19 minutes. Another finding revealed an almost complete concentration-dependent $\left(10^{2}-10^{7} \mathrm{CFU} / \mathrm{mL}\right)$ bacterial destruction following laser irradiation for 6-30 minutes. ${ }^{38}$

Monoclonal antibody-conjugated popcorn-shaped gold nanotechnology was employed for selective killing of S. typhimurium DT104 resistant to ampicillin, chloramphenicol, streptomycin, sulfonamides, and tetracycline. Following exposure to $670 \mathrm{~nm}$ laser $\left(200 \mathrm{~mW} / \mathrm{cm}^{2}\right)$, tryptic soy agar was used to assess complete killing of the bacteria. ${ }^{39}$

\section{Nano-photothermal therapy mediated by GNPs in Enterococcus faecalis MDR bacteria}

Another conjugate based on a catheter coating containing gold nanoshells (AuNSs) functionalized with carboxylate-terminated organosulfur compounds was developed ${ }^{40}$ to assess the antimicrobial effect against $E$. faecalis. The gold standards for nanoparticle characterization, transmission electron microscopy, and scanning electron microscopy, were used for imaging the morphology of AuNSs. Energy-dispersive $\mathrm{X}$-ray spectroscopy and X-ray photoelectron spectroscopy were used to detect and map their components and analyze their surface chemistry. Also, ultraviolet-vis spectroscopy and infrared thermography helped quantify the optical and thermal properties of the conjugate. The time-exposure ratio that would cause killing of bacteria subjected to heat 
was assessed following treatment with an infrared $(810 \mathrm{~nm})$ diode laser using different exposure times. Conversely, the clonogenic assay was used to assess the survival of the bacteria after the inoculation of AuNPs conjugates, in either the presence or absence of NIR radiation. When compared to controls, killing of $E$. faecalis occurred to a greater extent after 5 minutes of treatment with NIR radiation, and this increased even more significantly after 10 minutes. In conclusion, efficient killing of E. faecalis adherent to silicone can be induced by irradiated AuNPs conjugates.

In another study, ${ }^{41}$ antibody-conjugated oval-shaped gold nanotechnology was employed for selective targeting and killing of pathogenic bacteria. Synthesis of nanoparticles using bacteria followed by NIR radiation induced the photothermal lysis of pathogenic bacteria, which subsequently reduced cell viability.

\section{Nano-photothermal therapy mediated by GNPs in E. coli MDR bacteria}

GNR-covered kanamycin-loaded hollow $\mathrm{SiO}_{2}$ (HSKAu rod) nanocapsules were developed for drug delivery and photothermal killing of bacteria, demonstrating that the association between a chemical drug, physical sterilization, and the physical damage of biological systems can make the sterilization more effective and deliver the expected results. ${ }^{42}$ $\mathrm{SiO}_{2}$ nanocapsules loaded with kanamycin and covered with GNRs were incubated with E. coli BL21 and exposed to NIR laser irradiation for 20 minutes. The treatment resulted in an overall rate of sterilization of $53.47 \%$, and $34.49 \%$ for the individual components of the hybrid bactericidal material (carrier, chemical sterilization of kanamycin, and physical sterilization induced by NIR laser-excited GNRs). ${ }^{42}$ As they proved to accumulate on bacterial surfaces and possess twophoton-induced photoluminescence properties, positively charged gold nanospheres were used against bacteria by laser pulses with a duration recorded on the femtosecond time scale. Whether incubated or not with gold nanospheres, bacterial cells were subjected to femtosecond laser pulse irradiation with a power of $15 \mathrm{~mW}$, at $820 \mathrm{~nm}$, using the raster scan method and $8 \mathrm{~ms}$ dwell time. The Live/Dead cell viability assay was used to assess the killing effect triggered by these conjugates. SYTO 9 green fluorescent nucleic acid stain labeled all bacterial cells, while propidium iodide red fluorescent counterstain labeled compromised bacterial cells. When $S$. aureus or E. coli was not treated with gold nanospheres, no significant numbers of bacteria exhibited red fluorescence and were considered as dead cells, as the intact bacterial cell wall kept the red fluorescent stain from bursting in. The extent of bacterial killing was not considerable in these bacteria following irradiation. Treatment with gold nanospheres $\left(7.5 \times 10^{-12} \mathrm{M}\right)$ and radiation therapy enhanced bacterial killing. One treatment was enough to cause $88.8 \%$ antibacterial effects against $S$. aureus and $82.6 \%$ against $E$. coli.

In another study, ${ }^{43}$ the development of a photon-tothermal conversion nanosystem and exposure of GNRs to laser irradiation produced a quick increase in temperature in the media. Laser-excited GNRs were expected to cause photothermal lysis of the bacteria, which was examined by synthesizing GNRs (aspect ratio 3:4) and determining their optical and thermal properties. Short GNRs proved to absorb laser pulses ( $808 \mathrm{~nm}, 450 \mathrm{~mW}$ ) to a greater extent than long GNRs, and temperature was significantly higher in glass vials. When induced by continuous wave laser irradiation, these nanorods were able to increase the temperature to above $100^{\circ} \mathrm{C}$ in bulk media; thus, E. coli cell lysis was obtained by exposure of the bacteria-containing culture to NIR laser irradiation. $^{43}$

Huang et al reported the synthesis of polygonal GNPs with affinity for absorbing NIR light, using a photochemical reaction. ${ }^{24}$ Vancomycin, an antibiotic that binds the D-alanine (D-Ala) residues, was attached to the GNPs and the resulting construct was used for growth inhibition of pathogenic bacteria under NIR irradiation at $808 \mathrm{~nm}$. The conjugate was also able to recognize gram-positive and gramnegative cell walls, as indicated by transmission electron microscopy (Figure 5). Nonetheless, the formation of bonds between unmodified GNPs and these bacteria was absent. GNPs were able to destroy $>99 \%$ of bacteria $(S$. aureus, Streptococcus pyogenes, E. coli, MRSA) in 5 minutes under NIR irradiation as a result of the photothermal effect of this type of therapy. ${ }^{24}$

\section{Conclusion}

Although proof-of-principle experiments in which laser thermal ablation of MDR bacteria using bio-functionalized nanoparticles are reported in literature, yet, there is a need for further in vitro and in vivo, investigations. The potential for translation to human therapies is high, especially because GNPs exhibit low toxicity and chemical inertia.

This review shows that gold nanomaterials can be successfully used for photothermal treatment, paving the way for future materials with strong and selective biocide effects determined by combining colloid antibodies and strategies that induce cell killing, with possible applications in novel 

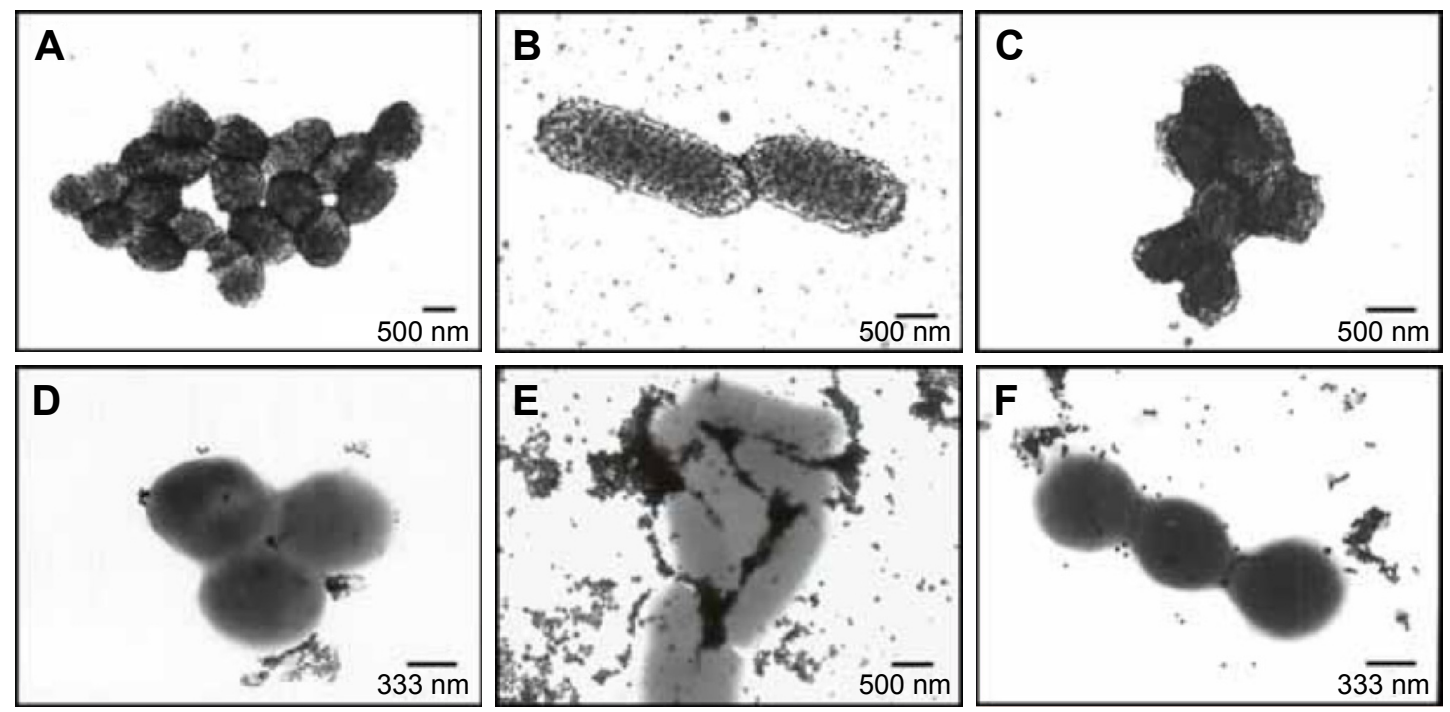

Figure 5 TEM images of (A) VRE, (B) PDRAB, and (C) VRSA produced after incubation with Au@van in PBS. TEM images of (D) VRE, (E) PDRAB, and (F) MRSA produced after incubation with unmodified gold nanoparticles in PBS.

Notes: Copyright @ 2007. Future Medicine Ltd. Reproduced from Huang W, Tsai P, Chen Y. Functional gold nanoparticles as photothermal agents for selective-killing of pathogenic bacteria. Nanomedicine. 2007;2(6):777-787..$^{24}$

Abbreviations: MRSA, methicillin-resistant Staphylococcus aureus; PBS, phosphate-buffered saline; PDRAB, pandrug-resistant Acinetobacter baumannii; TEM, transmission electron microscopy; VRE, vancomycin-resistant enterococci; VRSA, vancomycin-resistant Staphylococcus aureus.

therapies against MDR bacteria. The new materials based on a chemical drug associated with physical sterilization trigger a synergistic effect and increase the effectiveness of sterilization, and are thus promising candidates for adjuvant antibacterial treatment and disease control.

\section{Acknowledgments}

The authors would like to thank Mr Cosmin Puia for his support in gathering data enounced in this manuscript.

This work was supported by the Romanian National Authority for Scientific Research and Innovation, CNCS-UEFISCDI, project numbers PN-II-RU-TE-2014-4-2267; PN-IIPTPCCA-2011-3.1-1586; PN-II-PT-PCCA-2011-3.1-1551; PN-II-PT-PCCA-2011-3.2-1289;PN-II-PT-PCCA- 20134-1553;PN-III- P2-2.1-BG-2016- 0446; PN-III-P2-2.1-PED2016-1742; 4995/13/08.03.2016.

\section{Author contributions}

All authors contributed equally to this paper; made substantial contributions to conception and design, acquisition of data, or analysis and interpretation of data; took part in drafting the article or revising it critically for important intellectual content; gave final approval of the version to be published; and agree to be accountable for all aspects of the work.

\section{Disclosure}

The authors report no conflicts of interest in this work.

\section{References}

1. Giedraitienė A, Vitkauskienė A, Naginienė R, Pavilonis A. Antibiotic resistance mechanisms of clinically important bacteria. Medicina (kaunas). 2011;47(13):137-146.

2. Li X, Robinson S, Gupta A, et al. Functional gold nanoparticles as potent antimicrobial agents against multi-drug-resistant bacteria. ACS Nano. 2014;8(10):10682-10686.

3. Miller KP, Wang L, Chen Y, Pellechia P, Benicewicz B, Decho AW. Engineering nanoparticles to silence bacterial communication. Front Microbiol. 2015;6:189.

4. Smith R, Coast J. The true cost of antimicrobial resistance. BMJ. 2013; 346:f1493.

5. Veerapandian M, Yun K. Functionalization of biomolecules on nanoparticles: specialized for antibacterial applications. Appl Microbiol Biotechnol. 2011;90(5):1655-1667.

6. Wang P, Zhao Y, Tian Y, Jiang X. Multiple strategies to activate gold nanoparticles as potent antibacterial agents. Nanomed. 2016;12(2): $527-528$.

7. Yuan P, Ding X, Guan Z, et al. Plasmon-coupled gold nanospheres for two-photon imaging and photoantibacterial activity. Adv Healthc Mater. 2015;4(5):674-678.

8. Laxminarayan R, Duse A, Wattal C. Antibiotic resistance - the need for global solutions. Lancet Infect Dis. 2013;13(12):1057-1098.

9. Rossolini GM, Arena F, Pecile P, Polini S. Update on the antibiotic resistance crisis. Curr Opin Pharmacol. 2014;18:56-60.

10. Spellberg B, Bartlett JG, Gilbert DN. The future of antibiotics and resistance. N Engl J Med. 2013;368(4):299-302.

11. Zhao Y, Jiang X. Multiple strategies to activate gold nanoparticles as antibiotics. Nanoscale. 2013;5(18):8340-8350.

12. England CG, Huang JS, James KT, Zhang G, Gobin AM, Frieboes HB. Detection of phosphatidylcholine-coated gold nanoparticles in orthotopic pancreatic adenocarcinoma using hyperspectral imaging. PloS One. 2015;10(6): 0129172

13. Ilie I, Ilie R, Mocan T, Bartos D, Mocan L. Influence of nanomaterials on stem cell differentiation: designing an appropriate nanobiointerface. Int J Nanomedicine. 2012;7:3011-3025.

14. Mocan T, Clichici S, Agoston-Coldea L, et al. Implications of oxidative stress mechanisms in toxicity of nanoparticles (review). Acta Physiol Hung. 2010;97(3):247-255. 
15. Huo S, Ma H, Huang K, et al. Superior penetration and retention behavior of $50 \mathrm{~nm}$ gold nanoparticles in tumors. Cancer Res. 2013; 73(1):319-330.

16. Lin M, Pei H, Yang F, Fan C, Zuo X. Applications of gold nanoparticles in the detection and identification of infectious diseases and biothreats. Adv Mater. 2013;25(25):3490-3496.

17. Mieszawska AJ, Mulder WJ, Fayad ZA, Cormode DP. Multifunctional gold nanoparticles for diagnosis and therapy of disease. Mol Pharm. 2013;10(3):831-847.

18. Mocan L, Ilie I, Matea C, et al. Surface plasmon resonance-induced photoactivation of gold nanoparticles as bactericidal agents against methicillin-resistant Staphylococcus aureus. Int J Nanomedicine. 2014; 9:1453-1461.

19. Mocan T, Matea C, Tabaran F, Iancu C, Orosan R, Mocan L. In vitro administration of gold nanoparticles functionalized with MUC-1 protein fragment generates anticancer vaccine response via macrophage activation and polarization mechanism. J Cancer. 2015;6(6):583.

20. Mocan T, Matea C, Tabaran FA, et al. Photothermal treatment of liver cancer with albumin-conjugated gold nanoparticles initiates Golgi Apparatus-ER dysfunction and caspase-3 apoptotic pathway activation by selective targeting of Gp60 receptor. Int J Nanomedicine. 2015;10:5435.

21. Rengan AK, Bukhari AB, Pradhan A, et al. In vivo analysis of biodegradable liposome gold nanoparticles as efficient agents for photothermal therapy of cancer. Nano Letters. 2015;15(2):842-848.

22. Zhang X. Gold nanoparticles: recent advances in the biomedical applications. Cell Biochem Biophys. 2015;72(3):771-775.

23. Bucharskaya A, Maslyakova G, Terentyuk G, et al. Towards effective photothermal/photodynamic treatment using plasmonic gold nanoparticles. Int J Mol Sci. 2016;17(8):1295.

24. Huang W, Tsai P, Chen Y. Functional gold nanoparticles as photothermal agents for selective-killing of pathogenic bacteria. Nanomedicine. 2007;2(6):777-787.

25. Pissuwan D, Cortie CH, Valenzuela SM, Cortie MB. Functionalised gold nanoparticles for controlling pathogenic bacteria. Trends Biotechnol. 2010;28(4):207-213.

26. Zhao Y, Tian Y, Cui Y, Liu W, Ma W, Jiang X. Small molecule-capped gold nanoparticles as potent antibacterial agents that target gramnegative bacteria. J Am Chem Soc. 2010;132(35):12349-12356.

27. Zharov VP, Mercer KE, Galitovskaya EN, Smeltzer MS. Photothermal nanotherapeutics and nanodiagnostics for selective killing of bacteria targeted with gold nanoparticles. Biophys J. 2006;90(2):619-627.

28. Millenbaugh NJ, Baskin JB, DeSilva MN, Elliot WR, Glickman RD. Photothermal killing of Staphylococcus aureus using antibody-targeted gold nanoparticles. Int J Nanomedicine. 2015;10:1953-1960.

29. Meeker DG, Jenkins SV, Miller EK, et al. Synergistic photothermal and antibiotic killing of biofilm-associated Staphylococcus aureus using targeted antibiotic-loaded gold nanoconstructs. ACS Infect Dis. 2016; 2(4):241-250.
30. Shokri R, Salouti M, Zanjani RS. Anti protein A antibody-gold nanorods conjugate: a targeting agent for selective killing of methicillin resistant Staphylococcus aureus using photothermal therapy method. J Microbiol. 2015;53(2):116-121.

31. Kuo WS, Chang CN, Chang YT, Yeh CS. Antimicrobial gold nanorods with dual-modality photodynamic inactivation and hyperthermia. Chem Commun (Camb). 2009;32:4853-4855.

32. Jo W, Kim MJ. Influence of the photothermal effect of a gold nanorod cluster on biofilm disinfection. Nanotechnology. 2013;24(19):195104.

33. Fasciani C, Silvero MJ, Anghel MA, Argüello GA, Becerra MC, Scaiano JC. Aspartame-stabilized gold-silver bimetallic biocompatible nanostructures with plasmonic photothermal properties, antibacterial activity, and long-term stability. J Am Chem Soc. 2014;136(50): 17394-17397.

34. Pallavicini P, Dona A, Taglietti A, et al. Self-assembled monolayers of gold nanostars: a convenient tool for near-IR photothermal biofilm eradication. Chem Commun (Camb). 2014;50(16):1969-1971.

35. Borovička J, Metheringham WJ, Madden LA, Walton CD, Stoyanov SD, Paunov VN. Photothermal colloid antibodies for shape-selective recognition and killing of microorganisms. J Am Chem Soc. 2013; 135(14):5282-5285.

36. Norman RS, Stone JW, Gole A, Murphy CJ, Sabo-Attwood TL. Targeted photothermal lysis of the pathogenic bacteria, pseudomonas aeruginosa, with gold nanorods. Nano lett. 2008;8(1):302-306.

37. Teng CP, Zhou T, Ye E, et al. Effective targeted photothermal ablation of multidrug resistant bacteria and their biofilms with NIR-absorbing gold nanocrosses. Adv Healthc Mater. 2016;5(16):2122-2130.

38. Lin Y, Hamme AT 2nd. Gold nanoparticle labeling based ICP-MS detection/measurement of bacteria, and their quantitative photothermal destruction. J Mater Chem B Mater Biol Med. 2015;3(17):3573-3582.

39. Khan SA, Singh AK, Senapati D, Fan Z, Ray PC. Bio-conjugated popcorn shaped gold nanoparticles for targeted photothermal killing of multiple drug resistant Salmonella DT104. J Mater Chem. 2011; 21(44):17705-17709.

40. Khantamat $\mathrm{O}, \mathrm{Li} \mathrm{C}, \mathrm{Yu} \mathrm{F}$, et al. Gold nanoshell-decorated silicone surfaces for the near-infrared (NIR) photothermal destruction of the pathogenic bacterium E. faecalis. ACS Appl Mater Interfaces. 2015; 7(7):3981-3993.

41. Wang S, Singh AK, Senapati D, Neely A, Yu H, Ray PC. Rapid colorimetric identification and targeted photothermal lysis of Salmonella bacteria by using bioconjugated oval-shaped gold nanoparticles. Chemistry. 2010;16(19):5600-5606.

42. Hu B, Zhang L, Chen X, Wang J. Gold nanorod-covered kanamycinloaded hollow SiO 2 (HSKAu rod) nanocapsules for drug delivery and photothermal therapy on bacteria. Nanoscale. 2013;5(1):246-252.

43. Kim C, Yi DK, Kim PSS, Lee W, Kim MJ. Rapid photothermal lysis of the pathogenic bacteria, Escherichia coli using synthesis of gold nanorods. J Nanosci Nanotechnol. 2009;9(5):2841-2845.
International Journal of Nanomedicine

\section{Publish your work in this journal}

The International Journal of Nanomedicine is an international, peerreviewed journal focusing on the application of nanotechnology in diagnostics, therapeutics, and drug delivery systems throughout the biomedical field. This journal is indexed on PubMed Central, MedLine, CAS, SciSearch $®$, Current Contents $\AA /$ Clinical Medicine,

\section{Dovepress}

Journal Citation Reports/Science Edition, EMBase, Scopus and the Elsevier Bibliographic databases. The manuscript management system is completely online and includes a very quick and fair peer-review system, which is all easy to use. Visit http://www.dovepress.com/ testimonials.php to read real quotes from published authors. 